
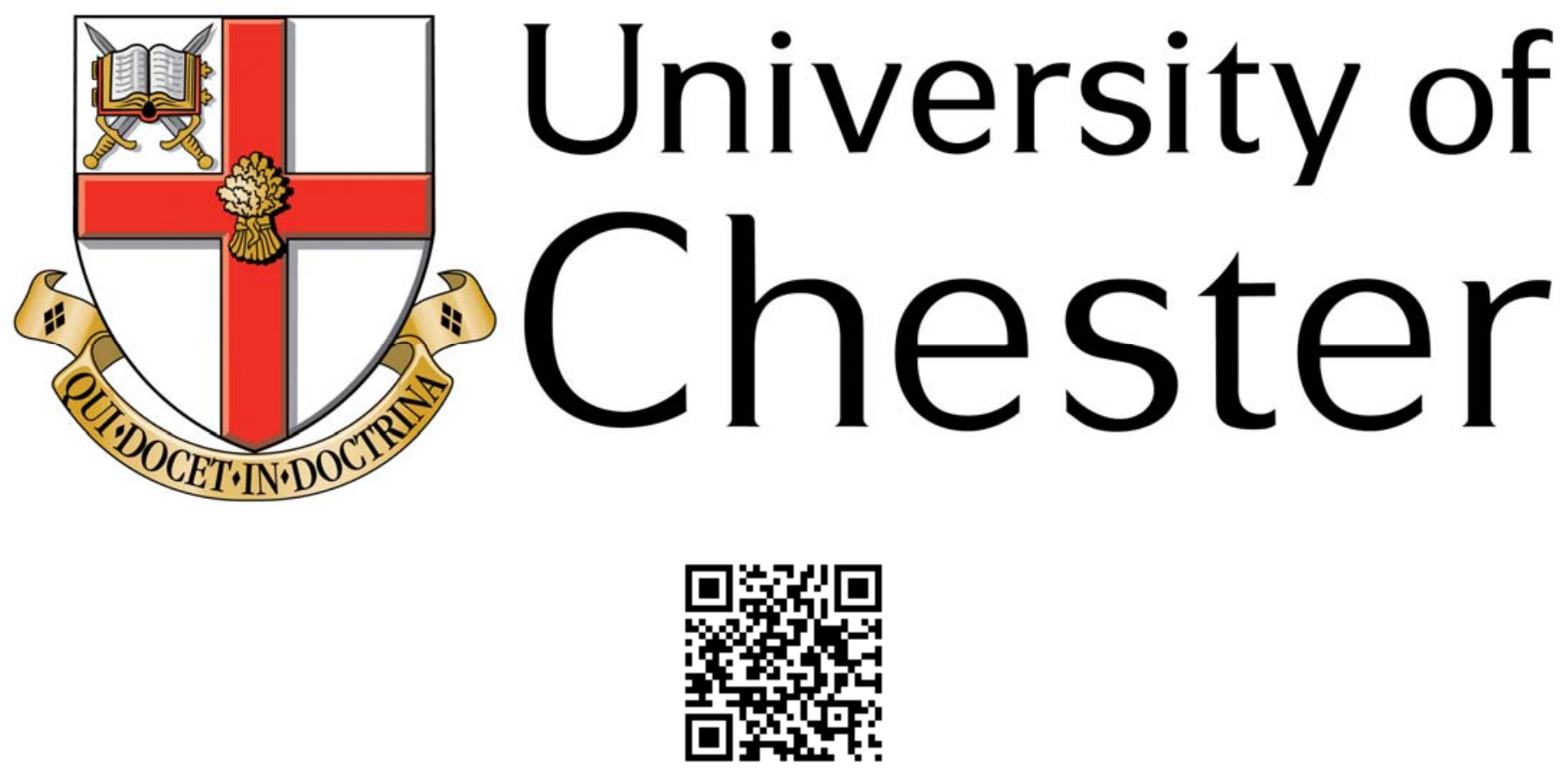

This work has been submitted to ChesterRep - the University of Chester's online research repository

\title{
http://chesterrep.openrepository.com
}

Author(s): Grzegorz Stefan Bankosz ; John Kerrins

Title: Mobile technology-enhanced asset maintenance in an SME

Date: 2014

Originally published in: Journal of Quality in Maintenance Engineering

Example citation: Bankosz, G. S., \& Kerrins, J. (2014). Mobile technology-enhanced asset maintenance in an SME. Journal of Quality in Maintenance Engineering, 20(2), 163-181

Version of item: Authors' post-print

Available at: http://hdl.handle.net/10034/316655 


\section{Mobile Technology-Enhanced Asset Maintenance in an SME}

\section{Introduction}

The increasing use of mobile technology is impacting on manufacturing just as it is across many spheres of activity. This paper presents an overview of how mobile devices are being integrated into a developing range of computing and communication technologies aimed at improving maintenance processes in manufacturing environments. The significance of effective maintenance from a business perspective underpins much of the discussion about advances in maintenance practice. While many of the initiatives focus on large scale developments, this paper illustrates how the emergence of mobile technology has provided an opportunity to build a demonstration prototype reflecting the agility and potential improvement in maintenance reporting that can be realised in much smaller less well-resourced environments.

We report on the development of a mobile solution whose initial motivation was to improve fault reporting in a small food manufacturing environment. We also demonstrate that the system begins to offer much richer potential for data acquisition, analysis and decision making. This is consistent with evolving ideas of e-maintenance, which we discuss to put our work into context. Our prototype raises interesting questions about the extent to which the use of powerful IT resources in larger organisations can inform flexibility and efficiency enhancements in small manufacturing environments. We also acknowledge the potential complexities of systems integration and the potential risks inherent in the deployment of emerging mobile technologies.

\section{Maintenance in Manufacturing}

The significance of asset maintenance in manufacturing is attested by a number of authors. Smith (2003) observes that an effective maintenance schedule enhances productivity and reliability, and reduces costs. In proposing a wireless-enabled environment integrating intelligent sensors transmitting data to a diagnostic centre handling system models supported by a domain knowledge base to facilitate maintenance processes, Arniaz, Emmanouilidis, Iung, \& Jantunen (2006) acknowledge the importance of maintenance strategies in ensuring productivity, quality standards and customer satisfaction. Improved efficiencies promote significant cost savings by reducing component failure and associated unscheduled shutdowns. The deployment of intelligent and webbased software has advanced the capability of these automated systems, which are now set to be enhanced by mobile access to data and resources. 


\subsection{Intelligent Maintenance}

The notion of e-maintenance is defined as a maintenance management concept in which assets are monitored and managed over the internet (Campos, Jantunen, \& Prakash, 2009). It is linked to advances in Condition Based Maintenance (CBM) in which web-based software agents (Wooldridge, 2009) are used in the monitoring and maintenance of mechanical and electrical systems. These data are then used for fault diagnosis and prognosis (Campos, 2009). The idea of real-time monitoring, control and optimisation of assets by the deployment of integrated intelligent software throughout the enterprise is discussed by US Department of Commerce (2011) who also recognise the challenges of establishing standards for data formats, devices and equipment to realise their goal.

Campos (2009) sees the emergence of web and agent technologies as underpinning an integrated concept of e-business, e-manufacturing and e-maintenance. The use of intelligent software is also seen as a potential means of capturing and representing knowledge and expertise. This idea is explored by Muller, Marquez, \& lung (2008) who posit the view that e-maintenance platforms can be augmented by the notion of e-collaboration in which enterprise integration encompasses suppliers, designers, process engineers and customers as a means of making organisational knowledge and intelligence available to support maintenance decisions. It is argued that analysis of information flows from e-maintenance platforms can contribute to corporate memory representing organisational experience in specific domains. The theme is also addressed by Campos, Jantunen, \& Prakash (2009) who assert that agent-based monitoring of asset data can be used for decision support and that this may be beneficial for users who have limited knowledge of the maintenance domain. The idea of capturing and reusing organisational knowledge is also considered by Gehman (2009) who suggests that data derived from low frequency maintenance incidents can be codified in suitable form such as decision trees which can be made available to staff via mobile devices and thus help detect potential malfunction before becoming critical.

\subsection{Impact of Mobility}

The advent of mobile technology has impacted hugely on many aspects of contemporary life and a number of authors assess its developing role in manufacturing and in maintenance. The potential benefits are acknowledged in terms of productivity, reliability and cost (Smith, 2003) and in terms of collaboration (Strategic Growth Concepts, n.d.), and communication and access to real-time data (Bagadia, 2010). The adoption of mobile technology is seen to complement a vision of enterprisewide integration of IT components to enhance end-to-end manufacturing processes (Majumdar \& Szigeti, 2011). It can be seen to complement increased flexibility and quality improvement deriving from integrated manufacturing environments with decentralised control structures (Gunawardana, 2006). Similarly, Cordis (2005) see wireless technology as a key factor in helping facilitate the role of 
IT in driving Europe's manufacturing competence and competitiveness through increased agility, flexibility and productivity. Yee and Gaja's case study of a Mobile Computer Maintenance Management System identifies a range of benefits delivered by the technology: essentially, increased access to asset data led to improvements in the quality and comprehensiveness of information which supported more efficient maintenance (Yee \& Gaja, 2008). Significantly, the authors also identify practical issues such as developing a suitable interface to support input of field data and offering training on the use and benefits of utilising the system to enhance asset management. This issue is also addressed by Emmanouilidis et al. (2009) in their overview of trends in employing wireless solutions to engineering asset and maintenance management. Mobile technology also introduces a number of significant organisational challenges as we discuss in Section 2.4 .

The benefits of utilising mobile technology to enhance manufacturing operations are addressed by Automation.com (2008) who comment on generating an enriched repository of data from more timely, accurate, mobile data collection which is then available for analysis and potentially more effective decision making. The importance of user engagement is also stressed if mobile devices are to be used fully to capture organisational data which then impacts on maintenance, safety and quality standards. Mobile devices are also seen as two-way channels facilitating access to organisational resources such as on-line manuals and decision support or productivity enhancement tools for example. Useful insight into the way smart phones are being used to access real-time engineering data is provided by (Bedder, 2011); and Bolukbasi (2011) comments on the flexibility of mobile applications in supporting manufacturing maintenance.

\subsection{Data Interoperability and Services Integration}

To fully realise the benefits of asset management, organisations need to integrate enterprise information. Jacobs \& Katz (2003) highlight the value of effective Enterprise Asset Management systems, particularly in co-ordinating information from disparate sources. The argument is presented by (Wilson, 2006) from a road hauliers' perspective where integration of enterprise systems can enable informed maintenance management decisions. The ability to ensure up to date enterprise information is seen to be beneficial across organisations in this domain irrespective of size. Emmanoulidis, Liyanage, \& Jantunen (2009) also stress the point that reliable and timely exchange of data between operations and maintenance and strategic management levels of an organisation underpins maintenance-related decisions. The authors identify the range of asset information inherent in the maintenance of industrial facilities and they highlight the importance of data integration in this process, particularly in view of the potential impact of mobile and wireless technologies in asset maintenance. 
In fact, the huge increase in data volumes and the shift towards data-intensive activity is acknowledged by (Bietz, Handel, Wiggins, \& Aragon, 2012) to be a developing phenomenon across science and engineering. Establishing standards to facilitate interoperability is seen as a key factor in enabling data interchange between different organisations, disciplines and technologies, but this is difficult to achieve.

The challenges posed by the need to establish effective interaction between heterogeneous systems are widely recognised and a number of authors have addressed issues of interoperability in different domains. They aim to develop standards and ontologies designed to specify core abstractions in specific domains.

Rajpathak \& Chougule (2011) acknowledge that data inconsistency and data mismatches have a critical impact on interoperability in distributed business environments. They express a need for a shared information model describing entities and abstractions existing in a particular domain. The entities' properties and the relations that exist between them are represented in an ontology which serves as a semantic model of the domain. Although ontology engineering is in its infancy in manufacturing and operational management, the authors posit a generic framework based on existing top-down knowledge representation techniques with partially automated bottom-up methods to extract and standardise conceptual terms from enterprise repositories. These are formulated into a class-based hierarchical ontology which is shown to improve the accuracy of data analysis in decision support within the automotive industry.

Similar issues underlie the difficulties in translating data between different computer aided design (CAD) formats. Tessier \& Wang (2013) propose an ontology representing key geometric and topological features that support mapping and verification capabilities for interoperability. These abstractions, requiring as little knowledge of source systems as possible, are represented in the Web Ontology Language (OWL) (OWL Web Ontology Language Current Status, 2007) which is integrated with the Semantic Web Rule Language (SWRL) (Horrocks, Patel-Schneider, Boley, Tabet, Grosof, \& Dean, 2004) to support automated reasoning.

The idea of standard representation to support interoperability also motivates the Core Manufacturing Simulation Data (CMSD) specification: a standardised, computer-interpretable formalism enabling efficient exchange of manufacturing shop-floor-related data for the creation and execution of manufacturing simulations (Yung-Tsun, Riddick, \& Johansson, 2011). Improved data interoperability can reduce simulation costs and improve the efficiency of modelling changes in the physical environment. As with (Tessier \& Wang, 2013), CMSD defines representations for essential 
manufacturing entities as neutral structures supporting efficient data exchange. The proposed framework, which builds upon existing approaches to standardised data integration, ISA-95 (ISA-95, 2010) and OAGi (Open Applications Group, 1996), is XML-based and supports web services.

Chou \& Seng (2012) address the question of how international telecommunications corporations deal with the integration of electronic services that underpin current business processes. The authors characterise the situation in terms of potentially chaotic relationships between client-facing web services and the backend and legacy systems with which they interact. They propose an XMLbased model designed to link a web-based client environment with the diverse backend and legacy systems that support business processes.

Issues surrounding data interoperability and service integration are at the core of advances in emaintenance, as is made clear by Arniaz, et al. (2010) in identifying the range of web-based technologies, sensors, wireless communications and mobile components comprising maintenance management systems. The demands of openness, integration and collaboration with other services within the enterprise add further complexity to the e-maintenance environment.

Jantunen, Gilabert, Emmanoulidis, \& Adgar (2009) assert that a key objective of e-maintenance is to make maintenance-related information ubiquitously available; this is supported by environments in which assets can be monitored and managed over the Internet. The collection and exchange of dynamic, real-time maintenance information supports the utilisation of detailed asset, or production system, knowledge. The authors demonstrate that e-maintenance principles can be implemented in a research environment in which the integration of sensors and distributed intelligent web services can be used to support condition monitoring, health assessment, prognosis and decision-support. The successful integration and interoperability of data, services and physical communication is cited as critical to the project's success. This is achieved by the following approaches respectively: interoperable XML-based data exchange formats and maintenance-oriented data format definitions such as MIMOSA (MIMOSA, 1998); web services integration through resource-oriented, or RESTful (Rodriguez, 2008), services; network integration through wired and wireless communication protocols including Bluetooth at operational and WiFi at higher enterprise levels. While this particular solution successfully adopts standard representation to support interoperability, it is acknowledged that incompatibility across platforms remains a general problem for e-maintenance initiatives.

The aim to establish standard formalisms to support interoperability and integration across heterogeneous systems remains a challenging area of continuing investigation. The adoption of 
standard languages such as XML and the development of consistent ontologies offer potential foundations for robust frameworks that can be extended to mobile environments to facilitate the powerful and flexible asset maintenance resources that these technologies offer.

\subsection{Wider issues - BYOD and Security}

The pervasive use of mobile devices across business and leisure activities raises significant issues about the implementation of suitable environments to support organisational mobile platforms. The effects of the 'consumerisation of IT' are acknowledged by Wipro Technologies (2012) and by IBM (2012). The latter paper links this to a rising trend of Bring Your Own Device (BYOD) emerging from users' desire to enjoy the same functionality in business devices as in personal ones. These changes are affecting organisational culture in which central control over the selection, deployment and support of mobile devices is being eroded. The effects of these changes are characterised by a move towards more extensive mobile applications beyond voice, e-mail and calendar functionality.

These trends are not without their risks and a number of authors focus on the security threats posed by the deployment of mobile devices. While acknowledging the increased corporate agility that mobile technology brings, Symantec Corporation (2012) highlight inherent risks represented by lost devices, data leakage, unauthorised access to corporate resources and potential malware infection. Lane (2012) also considers the risks to manufacturers and identifies the threat posed by employees circumventing or disengaging security features on company sponsored devices. Similar issues are also identified by the Ponemon Institute (2012), while Manufacturing Business Technology (2012) identifies ways in which the proliferation of different device types can affect operational efficiency.

Having drawn attention to the risks of trends such as BYOD many authors suggest ways of managing the risks so that benefits of mobile and wireless technology can be harnessed to enhance organisational processes. Many emphasise the importance of developing a suitable strategy to anticipate and resolve potential threats.

\subsection{Organisational Strategy}

There is clear support for the adoption of mobile and wireless technologies within organisations. Lane (2012) recommends the implementation of a mobile strategy designed to transform the organisation rather than streamlining existing processes. The Ponemon Institute (2012) advises that a comprehensive mobile policy should be implemented which should clarify acceptable use of mobile devices. This should be complemented by employee education aimed at promoting risk awareness. These measures should be reinforced by the deployment of enabling technologies to detect and prevent data theft and malware threats. Similar themes are addressed by IBM (2012) who recommend that key security measures be implemented: limit network access to authorised 
and verified users; encrypt transmitted data; manage device configurations to comply with regulatory requirements; undertake remote wiping of devices if sensitive business data have been compromised. The paper also considers additional steps that might be taken which include making light, thin organisational applications accessible through an internal store and extending existing business applications to mobile devices. Policies should be modified to accommodate the changes and employees should be made aware of their roles and responsibilities such that mutual benefits are gained from the flexibility that mobility offers.

Having acknowledged the costs of inadequate security represented by direct financial expenses, data loss, brand damage and loss of customer trust, Symantec Corporation (2012) also recommend a strategic approach to mobile deployment. They advise an extension of current processes, policies and technologies applied to desktop and laptop machines to mobile platforms; organisations must plan for employees adding devices to the corporate network and these changes should be addressed legally, operationally and culturally. Equally, existing security practices including encryption and authentication policies should be extended to maintain corporate and regulatory compliance.

\section{$3 \quad$ Developing the Mobile Engineering Work Request System}

We aimed to develop a system to explore the scope for introducing mobile technology into existing maintenance monitoring procedures within a food manufacturing plant. Although part of a larger organisation, the plant was relatively small, with some 278 employees, and it provided an opportunity to evaluate the potential benefits that a mobile solution might bring. The subsidiary already had its own maintenance engineering department. Plant and equipment were maintained by the Ultrasys Computerised Maintenance Management System aimed at preventative maintenance, and Plant Fault Note, which focuses on plant faults.

The existing system was configured such that the Plant Fault Note maintenance software was accessible through three workstations to which not all staff members had access rights. Consequently, faults were mainly reported to the Quality Assurance Department to be logged on the system. These tended to represent system and installation faults. Machinery and equipment faults, on the other hand, were likely to be reported verbally to the Engineering Department. As there was no system dedicated to comprehensive maintenance reporting, there was a gap in effective communication between departments which increased the potential for omissions, misunderstandings and loss of productivity. These are common maintenance issues, as discussed in Section 2. 
In order to address these limitations, we developed a prototype Mobile Engineering Work Request System enabling staff to directly report any machinery or equipment faults as they encountered them. Staff would also be able to report signs of wear and tear preceding actual breakdown thus helping prevent downtime. We have already discussed the potential benefits of this kind of resource in terms of enhancing the timeliness and accuracy of data and thereby improving productivity and maintenance processes.

The solution was customised to meet the specific engineering maintenance requirements of the food manufacturing plant. This was entirely feasible as an experimental prototype and system evaluation suggests that it has potential for further development and implementation within the organisation, as we discuss later in Section 4. Our approach touches upon the interesting debate about the merits of large, installation-based versus path-based models for IT development discussed by Upton and McAfee (2000). Certainly our small-scale, incremental and flexible development, which adopts open standards, is consistent with a path-based approach in which staff members are closely involved in the emergence of new organisational systems.

\subsection{System Requirements}

The prototype was based on an existing system which was constrained by limited access and flexibility, as discussed in Section 3. The aim was to maintain existing functionality in a mobile environment and to introduce some data handling enhancements to illustrate additional benefits. The following key features were identified:

1. Intuitive, user-friendly interface with effective navigation to encourage user participation

2. Password protected user login to secure data access

3. Implementation of three user groups with access level protection: administrator; engineer; user

4. Principal functionality:

4.1. Report a fault

4.2. Amend a fault report

4.3. Check status of reported faults

4.4. Output graphical statistical display of fault reporting activity

4.5. Schedule an engineer to handle a particular fault

4.5.1.Browse individual engineer maintenance schedule

4.5.2. Output graphical statistical display of schedule activity

4.6. Manage user accounts

In addition, the system must support multiple connections to the server. 


\subsection{Development Platform}

System configuration comprised a client access application and a remote database offering web services. The mobile application required the Java Development Kit (JDK) Version 7 (Oracle, n.d.) and the Android Software Development Kit (SDK) (Google Inc, n.d.) which provides the Android operating system and a set of developer's tools. Android code draws upon Java libraries and generates byte code optimised for Android devices (Meier, 2010). Android is open source software based on the Linux operating system (The Linux Foundation, 2012) and it is specifically designed for mobile devices. Additional software included the Eclipse Integrated Development Environment (IDE) (The Eclipse Foundation, 2013), which supports application development. This is used in conjunction with the Android Development Tools (ADT) Plugin which integrates the Android SDK with the Eclipse IDE. For this development, Adobe Photoshop was used to design graphics for the user interface (Adobe Systems Inc., 2013). This environment was supported by a standard desktop computer running a Microsoft Windows 7 operating system (Microsoft, 2013). The system was tested on an HTC Desire mobile phone using an Android operating system.

The server computer was running Microsoft Windows Server 2008 R2 (Microsoft, 2013) and Internet Information Service Version 7 which hosted the database. The latter consisted of MySQL database software integrated with PHP scripting language which was used to write the web service code enabling the Android device to interact with the hosted database (Welling \& Thomson, 2009).

PHP and Android support data interchange in the form of Java Script Object Notation (JSON) (w3Schools.com, 2013a) using Hypertext Transfer Protocol (HTTP) using POST and GET requests (w3Schools, 2013b). This enables the mobile device to communicate with the MySQL database using encrypted passwords via PHP middleware.

\subsection{System Integration}

The integration and interoperability of different systems components posed a significant challenge in developing the Mobile Engineering Work Request system. This was achieved by implementing JSON, a text-based open standard for human readable data interchange format (w3Schools.com, 2013a). This was an effective solution because of its openness and wide support by the Android operating system, MySQL and PHP. It enabled the integration of data between the client application and the database via a middleware web service utilising POST and GET HTTP requests (w3Schools, 2013b).

Initially, attempts were made at a direct connection between the mobile application and the database but the Android operating system does not easily support Java Database Connectivity drivers (JDBC), and there are security and reliability issues with this approach (androidforums.com, 
2012). The implementation of a RESTful service (Rodriguez, 2008) was chosen to provide an effective communication platform for the system.

Finally, the physical connection between the mobile application and the database was achieved through the adoption of GPRS/3G and WiFi connection standards. It was also important for the project to provide the end-users with the ability to use corporate Wi-Fi and mobile $3 \mathrm{G}$ networks.

Due to the nature and limited time-scale of the project, the prototype is not integrated with existing company systems. However, potential integration is feasible and relatively easy to achieve by customising the RESTful PHP-based web service.

\subsection{End to End Data and Service Workflow}

The following points demonstrate end to end data and service work flow for generating and executing work requests.

\section{Logging into the system}

The system user logs into the application by inputting their username and password. The application encrypts the user details and then sends them via HTTP POST request to the web service which executes the query to authenticate the user. An appropriate query based on the request tag value is then executed to get the user details, including access level permission, from the database. Once the process has been completed the mobile application displays the main menu screen. Figure 1 presents the data and service workflow for logging into the system.

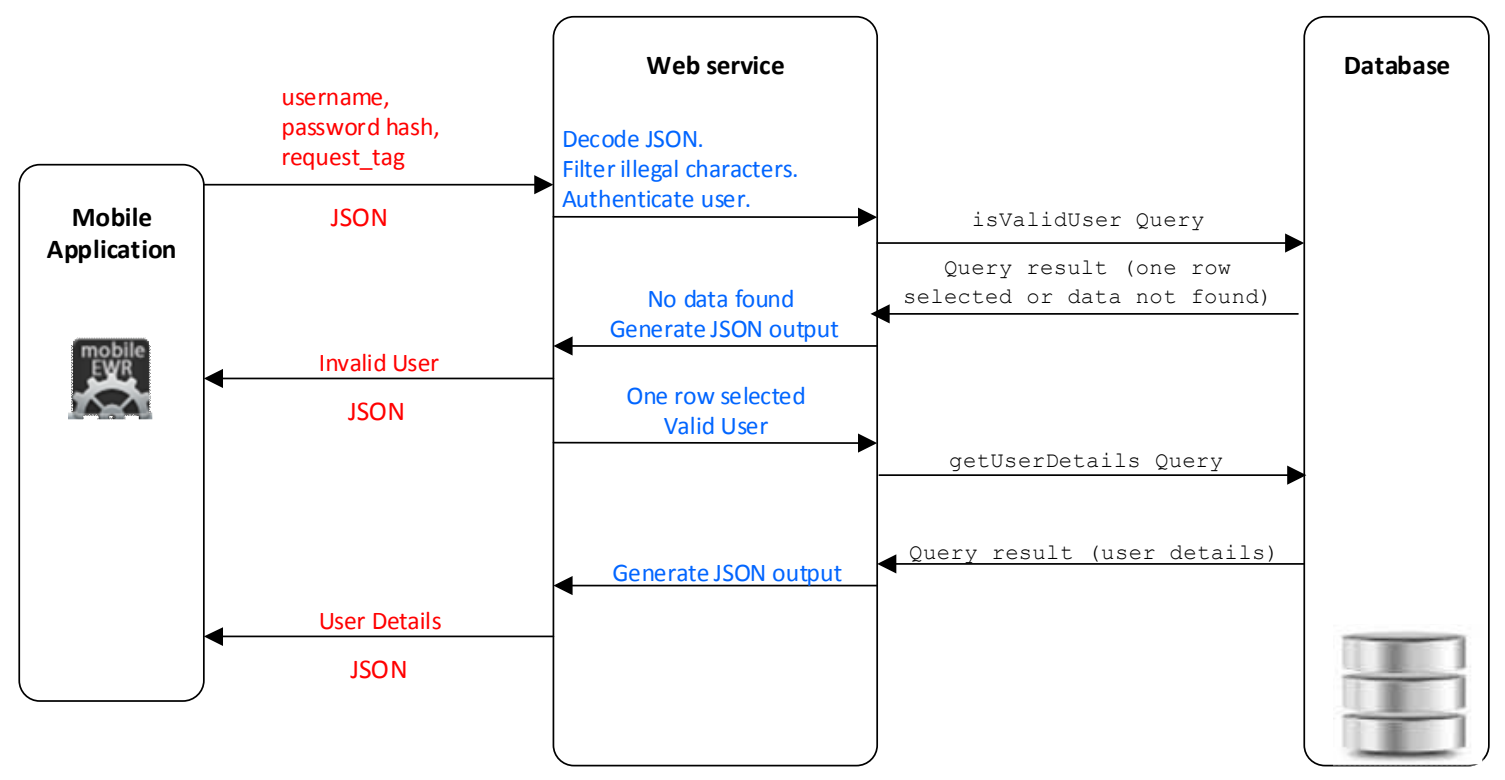

Figure 1 Logging into the System

\section{Reporting a fault within the plant or with equipment}


To report a fault, factory staff select the Report a Fault button from the main menu. Provided appropriate access level permission has been granted, the user is allowed to navigate to the Report Fault screen, where the following information can be input:

- $\quad$ Location [High Care / Low Care]

- $\quad$ Area [Line 1 ... Line 6]

- $\quad$ Priority [Urgent / Not Urgent]

- $\quad$ Fault at [Machinery / Plant]

- $\quad$ Description [Fault Description]

When all the details are completed the user selects the send button, which displays a confirmation message with all the recorded details. Once the confirmation button has been selected, the mobile application transfers the details together with the user details to the web service which performs data and user authentication checks. The web service executes the appropriate insert query, determined by the request tag value, and saves the details of the fault into the database. The web service returns the success or failure of the insert statement to the application, which displays the appropriate confirmation message. Figure 2 presents the data and service workflow for reporting a fault.

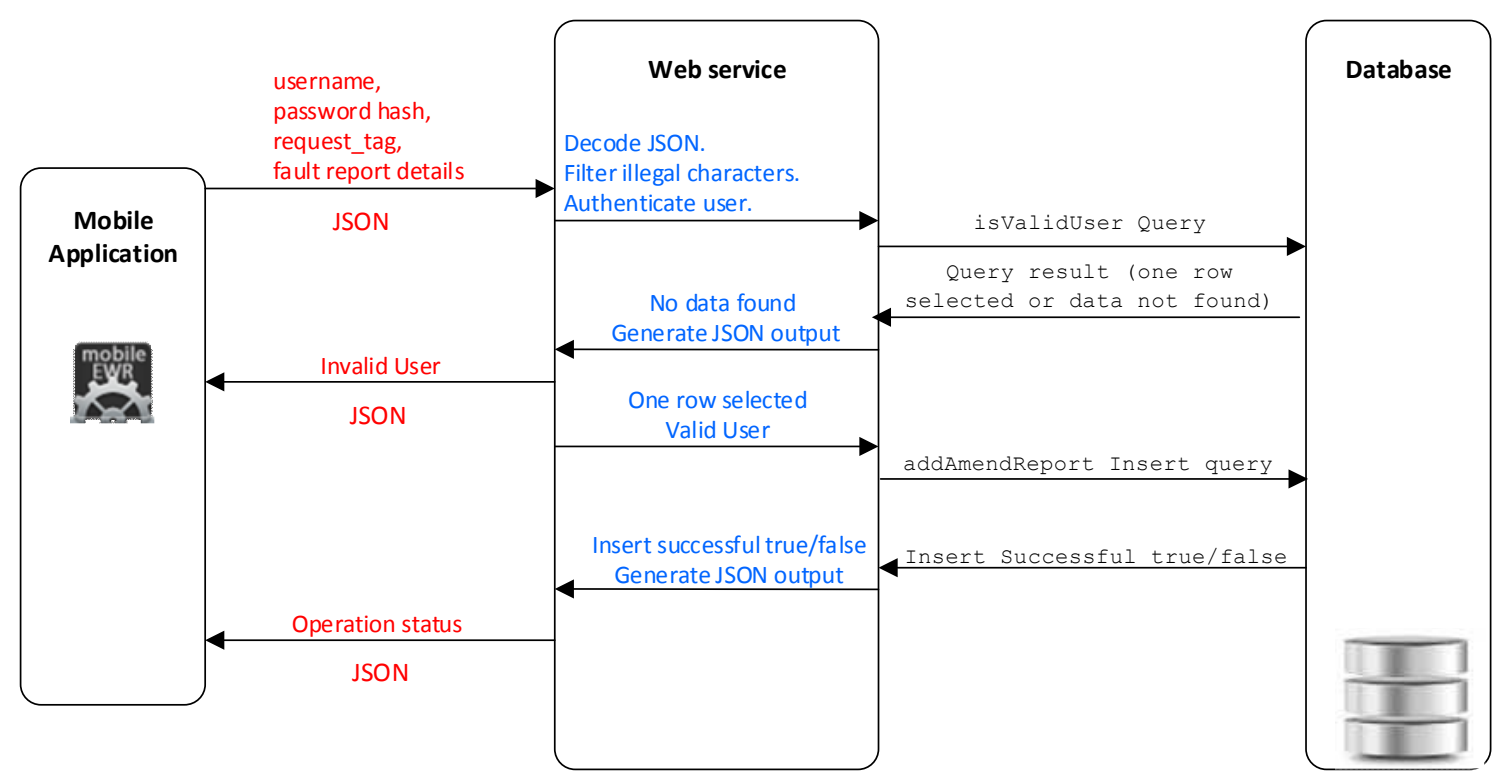

Figure 2 Reporting a Fault

\section{Scheduling an engineer}

After successful authentication, a member of the engineering management team selects the Assign Engineer button. The application checks the access level permission and sends a request to the web service to download the list of reported faults. The application displays the web 
service response in the form of a list. Next, the user selects a fault in order to assign the particular engineer to this task. This action triggers a request to return the list of available engineers for this particular task. The list is returned. An engineer is selected from the list to be scheduled to attend to the task. These details are returned by the mobile application which finally receives confirmation of the status of the query from the web service. Figure 3 presents the data and service workflow for scheduling an engineer.

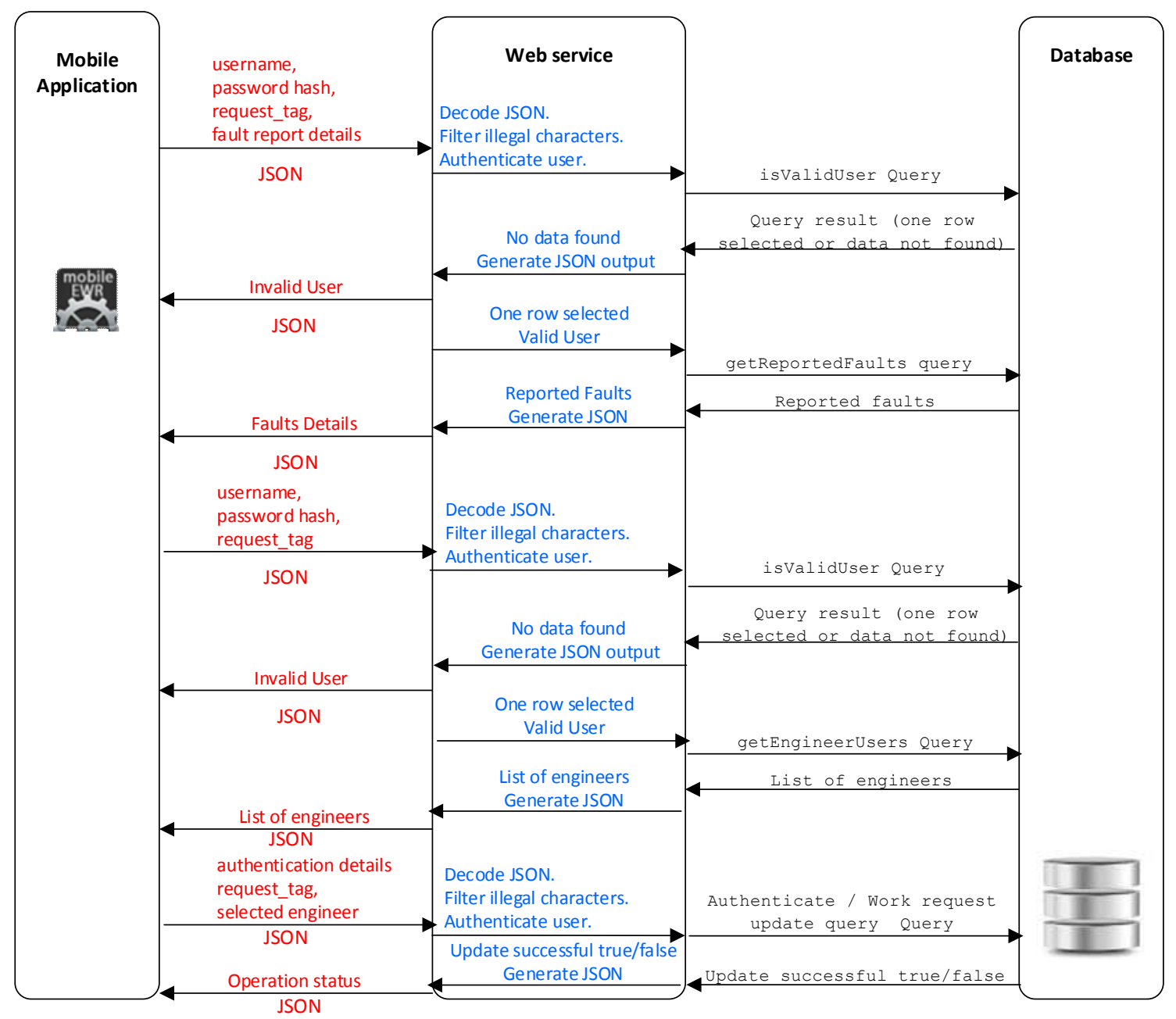

Figure 3 Scheduling an Engineer

\section{Completing a work request}

After successful authentication, the scheduled engineer selects the My Job button. The application checks whether the user has permission to access this section. The application sends the request for all jobs assigned to the logged-in engineer. The web service translates the request, authenticates the end user and queries the database. The mobile application displays the result in the form of a list. Once the job has successfully been completed, the engineer can select the job from the list and input a relevant comment, which is then submitted to the 
system. The web service translates the request and authenticates the user. Finally, the appropriate insert query based on the request tag value is executed and the resulting insert statement status message is sent back to the application, which then displays the confirmation message. Figure 4 presents the data and service workflow for completing a work request.

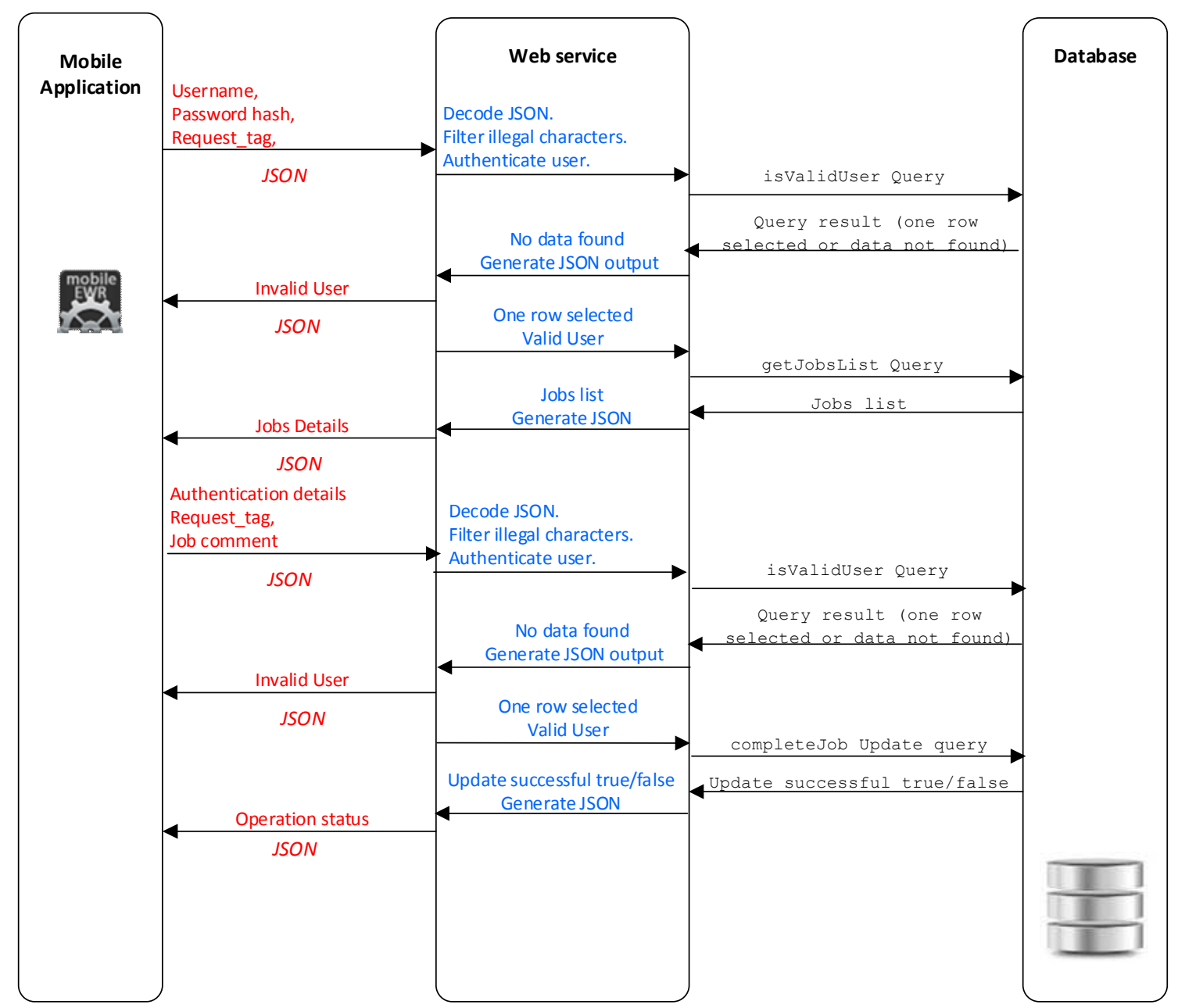

Figure 4 Completing a Work Request

\subsection{Sample Interfaces}

The following figures illustrate some of the system's functionality and convey a sense of the user experience with the smart phone interface. 

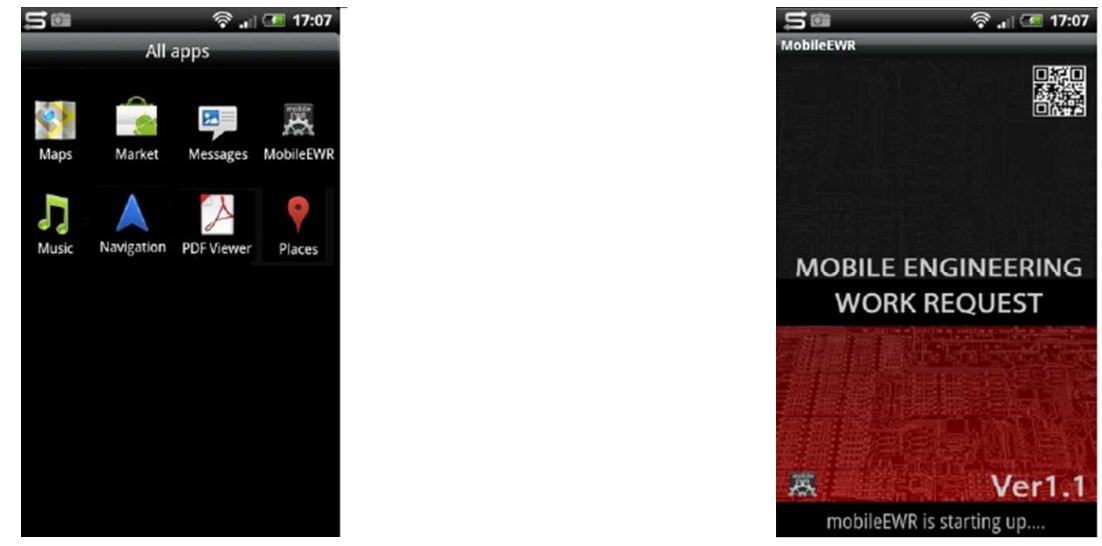

Figure 5 Mobile Engineering Work Request System Application Icon and Start-up Screen

Figure 5 illustrates a standard mobile phone interface from which applications are launched. The question of whether organisations should develop their own in-house mobile application store is raised by IBM (2012) as we discuss in Section 2.5. The start-up screen establishes a visual theme for the application.
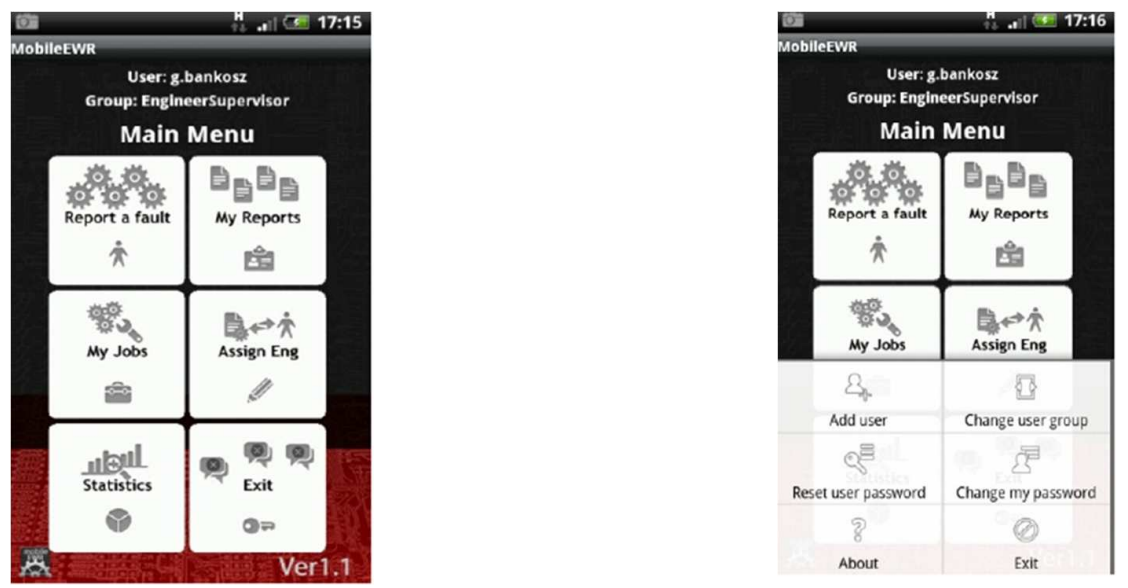

Figure 6 Main Menu and Administrator Options Screens

Figure 6 shows how a consistent interface theme has been developed. The Main Menu screen presents simple, intuitive graphics designed to aid navigation within the constraints of mobile interfaces. As Yee and Gaja (2008) caution (see Section 2.2), this kind of detail can impact upon the success of mobile initiatives. The administrator has basic system management privileges, as illustrated. 

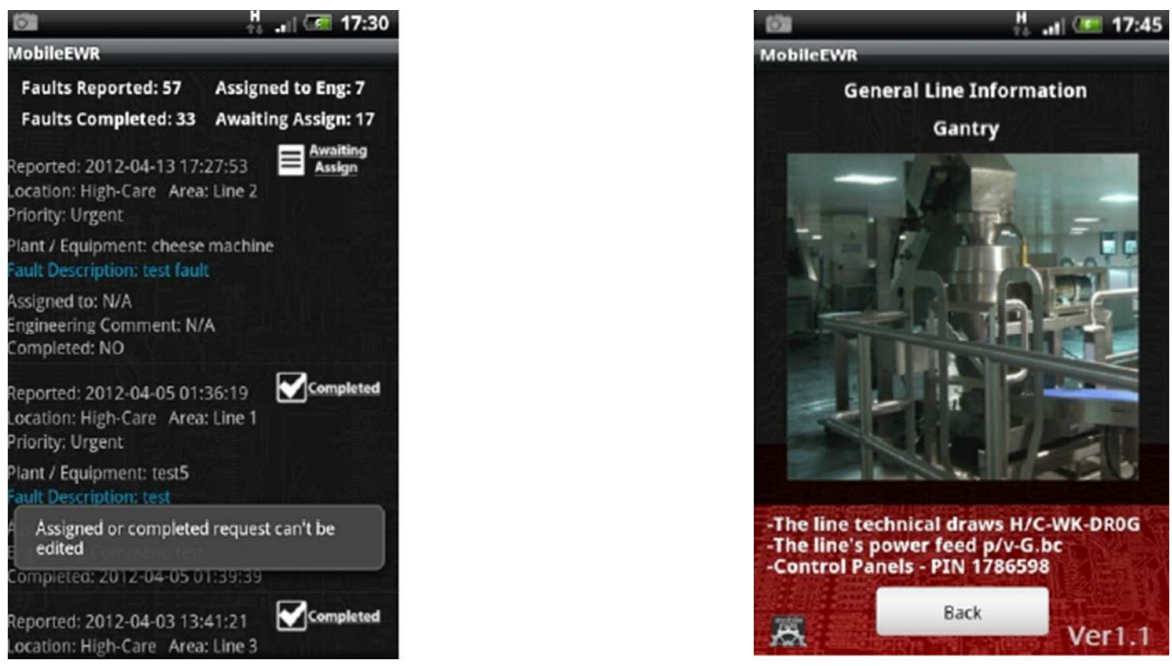

Figure 7 Functional Interaction

Figure 7 illustrates user interaction with the fault reporting systems and also demonstrates the potential for using images to clarify communication and to enhance the quality and effectiveness of maintenance information. The benefits of this kind of improved access to systems and information is discussed in Section 2.2.

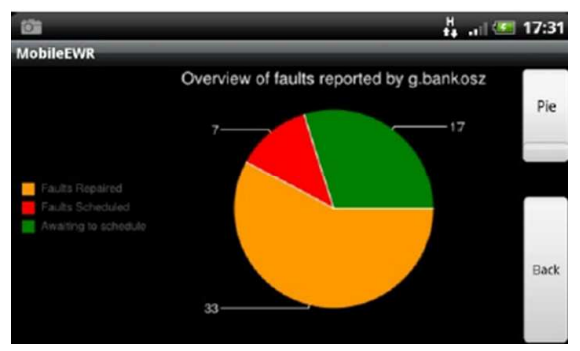

Figure 8 Graphical Representation

Figure 8 is indicative of the ways in which standard graphical representations can be used to summarise trends within the data. The utilisation of this type of resource has potential benefits for dissemination of information regarding performance indicators, for example, and can be used to aid decision making, as observed by Automation.com (2008).

\section{$4 \quad$ Organisational Evaluation}

Existing maintenance data were imported into the database so that relevant queries could be demonstrated using historical data. A small-scale evaluation of the system was carried out to get indicative feedback on its potential usefulness within the organisation. One of the respondents to the survey, the Engineering Manager, acknowledged the potential benefits in terms of improving 
both maintenance job allocation and the fault reporting mechanism, particularly the increased flexibility in view of the restricted PC access, and the potential value of using photographs to replace or enhance written descriptions. The manager also raised questions about the robustness of standard mobile phones in a factory environment - an issue also addressed by Wipro Technologies (2012) - and commented that the system might be more useful if it were integrated with the existing fault reporting software.

A technician from the engineering team expressed satisfaction with the system interface and functionality which made maintenance reporting and management simpler and quicker. These observations were reinforced by an acknowledgement that some faults went unreported while others came to light more informally. The ability to capture fault details in real time and to schedule and monitor maintenance activity directly offered scope for significant improvement in departmental communication and job prioritisation. Another technician who responded to the survey welcomed the application's functionality which was seen to offer significant potential for extending and enhancing the existing system.

Finally, from a senior management perspective, the Operations Director also identified the advantages of flexibility, the timeliness and accuracy of data, and improved communication as potentially worthwhile benefits. The statistical analysis feature was seen as potentially useful provided a clear rationale for pattern detection was formulated. Ideally, the Operations Director also wished to see the system integrated with existing software resources.

These comments from the evaluation survey are consistent with much of the debate in the literature, which we introduced earlier in this paper.

\subsection{Towards Implementation}

The aim of the project was to explore the potential benefits and limitations of introducing mobile and wireless technologies to the existing asset maintenance system. This was to be achieved by developing a prototype system to demonstrate key asset maintenance functionality. The initial scope did not extend to system implementation.

The positive feedback on the prototype elicited from various members of the organisation clearly indicates that there is recognition of the potential benefits of exploiting these new technologies. Moving towards a mobile architecture to support asset maintenance offers scope to improve asset monitoring and diagnosis (Emmanoulidis, Liyanage, \& Jantunen, 2009) and also decision support (Jantunen, Gilabert, Emmanoulidis, \& Adgar, 2009). The role of an efficient maintenance operation, supported by these technologies, can also impact critically on cost, quality and delivery performance (Jantunen, Gilabert, Emmanoulidis, \& Adgar, 2009). 
In the first instance, a feasibility analysis should evaluate the technical challenges of integrating mobile capability into the current system. This should identify the impact on business processes and estimate potential cost benefits (DeLone \& McLean, 2003), (Dennis, Wixom, \& Roth, 2010). While the return on investment may be significant if potential operational maintenance efficiencies are realised, issues concerning system development and integration and their attendant risks must be carefully addressed (Nicholas \& Steyn, 2012). Clearly, all stakeholders, including machine operators, maintenance engineers and managers must acknowledge potential system benefits if it is to be implemented successfully.

The new prototype provides a useful vehicle for clarifying system requirements (Laplante, 2009) and it is likely that an iterative prototyping approach, working closely with users, would be adopted in further system development (Rajlich, 2012). A parallel conversion strategy would be suitable when implementing the new system (Stair \& Reynolds, 2012). Although placing additional demands on staff (Dennis, Wixom, \& Roth, 2010), this retains the existing system, in case of any contingency, until thorough testing has been completed and the new system is ready for use.

A critical factor in migrating to a mobile, e-maintenance-focused environment is the question of device availability, e.g. BYOD strategy and the associated security issues, as discussed in Section 2.4. The capacity to specify and implement a sufficiently flexible and robust architecture to support the modified infrastructure should be evident in the technical feasibility analysis.

\section{$5 \quad$ Concluding Remarks}

This paper has reported on the development of an experimental prototype mobile application designed to improve fault maintenance procedures in a small food processing plant. We have presented the work within the wider context of attempts to improve the vital task of asset maintenance in manufacturing by utilising computing and communications resources. Our discussion has focused on attempts in large-scale environments to harness sophisticated software capability to monitor and predict resources' continued fitness for operation. By anticipating failure, downtime and attendant costs and loss of productivity can be ameliorated. The advent of mobile communication and interaction has brought a significant new dimension to these initiatives.

Our prototype has demonstrated that the introduction of mobile communications onto the factory floor offers the immediate possibility of improving the speed and accuracy of data capture as well as enhancing fault maintenance scheduling and enriching channels of communication between personnel engaged in the maintenance process. Apart from the technology, organisations also wish 
to involve staff in optimising business processes. By contributing to the effective management of organisational assets and recognising the consequent organisational benefits, they are more involved in achieving organisational aims. Evaluation feedback suggests that staff would be interested in contributing to the design of an upgraded system. This might attempt more ambitious automated analysis of maintenance data linked to graphical reports. Arguably, there are benefits in this in-house developmental approach (Upton \& McAfee, 2000) whereby staff may become more engaged in individual and collective development and contribute more fully to organisational knowledge and learning (Easterby-Smith \& Lyles, 2011), (Hislop, 2013) .

However, we must not gloss over the challenges that our development presents. Our prototype incorporates password encryption security measures but issues concerning data security on mobile devices must be addressed at a strategic level, as discussed in Section 2.5. Android devices have grown hugely in popularity over recent years and so provided a useful environment for development but the proliferation of device types and the implications of BYOD policies must also be carefully planned and managed, as we have acknowledged.

Our initiative has demonstrated the potential benefits of introducing mobile wireless technology and associated software into the asset maintenance facility of a small food manufacturing enterprise. Although there are technical challenges in scaling up the system, evidence from initial system testing suggests that some of the functionality and flexibility of automated e-maintenance could be established within this environment. This would help the organisation to continue to improve its maintenance processes and the development provides an insight into the kinds of approach that other SMEs might wish to consider.

\section{Bibliography}

Adobe Systems Inc. (2013). Adobe Photoshop. Retrieved April 19, 2013, from adobe.com: http://www.adobe.com/uk/products/photoshop.html

androidforums.com. (2012). Android + MySQL using com.mysql.jdbc.Driver. Retrieved November 01, 2013, from androidforums: http://androidforums.com/application-development/497414android-mysql-using-com-mysql-jdbc-driver.html

Arniaz, A., Emmanouilidis, C., lung, B., \& Jantunen, E. (2006). Mobile Maintenance Management. Journal of International Technology and Information Management, 15(4), 11-22.

Arniaz, A., lung, B., Adgar, A., Naks, T., Tohver, A., Tommingas, T., et al. (2010). Information and Communication Technologies within E-maintenance. In K. Holmberg, A. Adgar, A. Arniaz, E. Jantunen, J. Mascolo, \& S. Mekid (Eds.), E-maintenance (pp. 39-60). London: Springer.

Automation.com. (2008). How Mobile Technology can Optimize the Human Element in Manufacturing Operations. Automation.com. 
Bagadia, K. (2010, March). The Benefits and Challenges of Wireless CMMS. Retrieved April 16, 2013, from facilitiesnet:

www.facilitiesnet.com/mobile/ms/topicDetails.asp?id=11577\&topic=software

Bedder, S. (2011, November 24). How Mobile Technology can Drive Benefits for Manufacturers across the Design and Engineering Process. Retrieved April 18, 2013, from processindustryinformer.com: http://www.processindustryinformer.com/Editorial-FeatureArchive/How-Mobile-Technology-Can-Drive-Benefits-for-Manufacturers-across-the-Designand-Engineering-Process

Bietz, M. J., Handel, M., Wiggins, A., \& Aragon, C. (2012). Data-Intensive Collaboration in Science and Engineering. CSCW'12. Seattle: ACM.

Bolukbasi, H. (2011, September 23). Three Step Process for Mid-size Manufacturers to Implement Mobile Apps. Retrieved April 18, 2013, from industryweek.com: http://www.industryweek.com/information-technology/three-step-process-mid-sizedmanufacturers-implement-mobile-apps

Campos, J. (2009). Development in the application of ICT in condition monitoring and maintenance. Computers in Industry, 60, 1-20.

Campos, J., Jantunen, E., \& Prakash, O. (2009). A web and mobile device architecture for mobile emaintenance. International Journal of Advanced Manufacturing Technology, 45, 71-80.

Chou, T.-H., \& Seng, J.-L. (2012). Telecommunication e-services orchestration enabling business process management. Transactions on Emerging Telecommunications Technologies, 23, 649659.

Cordis. (2005, March). ICT for Manufacturing. Retrieved April 16, 2013, from cordis.europa.eu: ftp://ftp.cordis.europa.eu/pub/ist/docs/dir_g/ict-f-manuf_en.pdf

DeLone, W. D., \& McLean, E. R. (2003). The DeLone and McClean Model of Information Systems Success: A Ten-Year Update. Journal of Management Information Systems, 19(4), 9 - 30.

Dennis, A., Wixom, B. H., \& Roth, R. M. (2010). Systems Analysis and Design (4th Ed). Hoboken NJ: John Wiley \& Sons.

Easterby-Smith, M., \& Lyles, M. A. (2011). The Evolving Field of Organizational Learning and Knowledge Management. In M. Easterby-Smith, \& M. A. Lyles, Handbook of Organizational Learning \& Knowledge Management (2nd ed., pp. 1-20). Chichester: John Wiley \& Sons Ltd.

Emmanoulidis, C., Liyanage, J. P., \& Jantunen, E. (2009). Mobile solutions for engineering asset and maintenance management. Journal of Quality in Maintenance Engineering, 15(1), 92-105.

Gehman, D. (2009, October 2). Using Mobile Wireless Technology in Manufacturing. Retrieved April 16, 2013, from AutomationWorld: www.automationworld.com/asset-management/usingmobile-wireless-technology-manufacturing 
Google Inc. (n.d.). Get the Android SDK. Retrieved April 19, 2013, from developer.android.com: http://developer.android.com/sdk/index.html

Gunawardana, K. D. (2006). Introduction of Advanced Manufacturing Technology: a literature review. Sabaragamuwa University Journal, 6(1), 116-134.

Hislop, D. (2013). Knowledge Management in Organizations A Critical Introduction (3rd ed.). Oxford: Oxford University Press.

Horrocks, I., Patel-Schneider, P. F., Boley, H., Tabet, S., Grosof, B., \& Dean, M. (2004, 05 21). SWRL: A Semantic Web Rule Language. Retrieved November 20, 2013, from w3.org: http://www.w3.org/Submission/SWRL/

IBM. (2012, January 13). Mobility is moving fast. To stay in control, you have to prepare for change. Retrieved April 18, 2013, from ibm.com: $\mathrm{ftp} / / /$ public.dhe.ibm.com/common/ssi/ecm/en/tiw14132usen/TIW14132USEN.PDF

ISA-95. (2010). Welcome to ISA-95. Retrieved November 21, 2013, from ISA-95.com: www.isa95.com

Jacobs, D., \& Katz, J. (2003). Watch your assets. Transportation \& Distribution, 22-24.

Jantunen, E., Gilabert, E., Emmanoulidis, C., \& Adgar, A. (2009). E-Maintenance, a Means to High Overall Efficiency. 4th World Congress on Engineering Asset Management. Athens: Springer.

Lane, B. (2012, July 24). Manufacturing Goes Mobile. Retrieved April 18, 2013, from ThomasNet.com: http://news.thomasnet.com/IMT/2012/07/24/manufacturing-goesmobile/

Laplante, P. A. (2009). Requirements Engineering for Software and Systems. Boca Raton FL: Auerbach Publications.

Majumdar, A., \& Szigeti, H. (2011). The ActionPlanT Vision for Manufacturing 2.0. Retrieved April 16, 2013, from ActionPlantT Factories of the Future: www.actionplant-project.eu

Manufacturing Business Technology. (2012, January 19). mbtmag.com. Retrieved April 18, 2013, from Mobile Technology : A Means To Improve Operational Efficiency: http://www.mbtmag.com/articles/2012/01/mobile-technology-means-improve-operationalefficiency

Meier, R. (2010). Professional Android 2 Application Development (1st ed.). Indianapolis: Wiley.

Microsoft. (2013). Do more with Windows 7. Retrieved April 19, 2013, from microsoft.com: http://windows.microsoft.com/en-gb/windows7/get-know-windows-7

Microsoft. (2013). Server and Coud Platform. Retrieved April 19, 2013, from microsoft.com: http://www.microsoft.com/en-us/server-cloud/windows-server/ 
MIMOSA. (1998). MIMOSA An Operations and Maintenance Information Open Systems Alliance. Retrieved November 22, 2013, from MIMOSA An Operations and Maintenance Information Open Systems Alliance: www.mimosa.org

Muller, A., Marquez, A. C., \& lung, B. (2008). On the concept of e-maintenance: Review and current research. Reliability Engineering and System Safety, 93, 1165 - 1187.

Nicholas, J. M., \& Steyn, H. (2012). Project Management for Engineering, Business and Technology (4th Ed). Abingdon: Routledge.

Open Applications Group. (1996). Open Applications Group - Open Standards that Open Markets. Retrieved November 21, 2013, from OAGi: http://www.oagi.org/dnn2/

Oracle. (n.d.). Java Platform Standard Edition 7 Names and Versions. Retrieved April 19, 2013, from oracle.com: http://www.oracle.com/technetwork/java/javase/jdk7-naming-418744.html

Ponemon Institute. (2012, February). Global Study on Mobility Risks: United States. Retrieved April 18, 2013, from ponemon.org: http://www.ponemon.org/local/upload/file/Websense_Mobility_US_Final.pdf

Rajlich, V. (2012). Software Engineering The Current Practice. Boca Raton FL: CRC Press.

Rajpathak, D., \& Chougule, R. (2011). A generic ontology framework for data integration and decision support in a distributed environment. International Journal of Computer Integrated Manufacturing, 24(2), 154 - 170.

Rodriguez, A. (2008). RESTful Web Services: The Basics. Retrieved November 01, 2013, from IBM: http://www.ibm.com/developerworks/webservices/library/ws-restful/

Smith, R. (2003, January). Best Practices: Maximizing Maintenance. Retrieved April 16, 2013, from facilitiesnet: http://www.facilitiesnet.com/maintenanceoperations/article/Best-practicesMaximizing-maintenance--1858\#

Stair, R., \& Reynolds, G. (2012). Information Systems (10th Ed). Course Technology.

Strategic Growth Concepts. (n.d.). Mobile Technology for Increased Productivity \& Profitabiity. Retrieved April 16, 2013, from Strategic Growth Concepts:

http://www.strategicgrowthconcepts.com/growth/increase-productivity--profitability.html

Symantec Corporation. (2012). 2012 State of Mobility Survey : Global Findings. Retrieved April 18, 2013, from symantec.com:

http://www.symantec.com/en/uk/content/en/us/about/media/pdfs/bstate_of_mobility_survey_2012.en-us.pdf

Tessier, S., \& Wang, Y. (2013). Ontology-based feature mapping and verification between CAD systems. Advanced Engineering Informatics, 27, 76 - 92.

The Eclipse Foundation. (2013). About The Eclipse Foundation. Retrieved April 19, 2013, from eclipse.org: http://www.eclipse.org/org/ 
The Linux Foundation. (2012). Linux.com. Retrieved April 19, 2013, from linux.com: www.linux.com

Upton, D. M., \& McAfee, A. P. (2000). A path-based approach to information technology in manufacturing. International Journal of Technology Management, 20(3), 354-372.

US Department of Commerce. (2011). Smart Manufacturing and Construction Systems Program. Retrieved April 16, 2013, from National Institute of Standards and Technology: http://www.nist.gov/el/isd/cs/smanuconsys.cfm

W3C. (2007). OWL Web Ontology Language Current Status. Retrieved 11 20, 2013, from w3.org: http://www.w3.org/standards/techs/owl\#w3c_all

w3Schools. (2013b). w3Schools.com. Retrieved April 19, 2013, from TCP/IP Protocols: http://www.w3schools.com/tcpip/tcpip_protocols.asp

w3Schools.com. (2013a). JSON Tutorial. Retrieved April 19, 2013, from w3Schools.com: http://www.w3schools.com/json/

Welling, L., \& Thomson, L. (2009). PHP and MySQL Web Development (4th ed.). Upper Saddle River: Addison Wesley.

Wilson, D. (2006). EvenSmall Fleets Benefit from Maintenance Management Systems. Dairy Foods 107.2, 55.

Wipro Technologies. (2012, February). Mobility in Manufacturing: Optimizing Process and Productivity. Retrieved April 18, 2013, from wipro.com: http://www.wipro.com/Documents/Mobility_in_Manufacturing.pdf

Wooldridge, M. (2009). An Introduction to MultiAgent Systems (2nd ed.). Chichester: John Wiley \& Sons Ltd.

Yee, V., \& Gaja, N. (2008). Mobile CMMS solutions jump starts asset management. Proceedings of the Water Environment Federation, (pp. 1509-1520).

Yung-Tsun, T. L., Riddick, F. H., \& Johansson, B. J. (2011). Core Manufacturing Simulation Data - a manufacturing simulation integration standard: overview and case studies. International Journal of Computer Integrated Manufacturing, 24(8), 689 - 709. 